\section{Handle with care - interpretation, synthesis and dissemination of data on paracetamol in pregnancy}

\section{Per Damkier (1D, Brian Cleary, Corinna Weber-Schoendorfer, \\ Svetlana Shechtman, Matteo Cassina, Alice Panchaud and Orna Diav-Cirtin}

Nature Reviews Endocrinology recently published a Consensus Statement by Bauer et al. (Bauer, A.Z. et al. Paracetamol use during pregnancy - a call for precautionary action. Nat Rev Endocrinol. 17, 757-766 (2021) $\left.)^{1}\right)$. The 13 authors - who do not represent a collaborative effort on behalf of health authorities or medical speciality organizations - call for caution. They suggest that there is now sufficient evidence that in utero exposure to paracetamol (otherwise known as acetaminophen or $N$-acetyl- $p$-aminophenol (APAP)) alters fetal development to an extent that warrants clinical action. We recognize that the authors reflect appropriately upon the inconsistencies and limitations of the underlying data, and we support their call for better data.

We disagree with their assessment of existing data and their clinical conclusions.

On face value, the main clinical conclusion by the authors, "We recommend that APAP should be used by pregnant women cautiously at the lowest effective dose for the shortest possible time," can hardly be disagreed with. These conclusions are straightforward: nobody should use medications without appropriate consideration of indication, efficacy and adverse reactions. Nobody should use medications at higher doses or for longer durations than necessary.

Preceding these conclusions, the authors present their assessment of the underlying evidence and argue that APAP use during pregnancy comes with non-trivial and disturbing risks to the fetus, and long-term developmental risks. For example: “Together, increasing evidence suggest that APAP is associated with male urogenital and reproductive tract abnormalities," and "...we believe the combined weight of animal and human evidence is strong enough for pregnant women to be cautioned." Here, we outline the principal methodological issues, and challenge the presentation and interpretation of specific studies.

First, the authors draw clinical conclusions using data from which causality cannot be effortlessly inferred ${ }^{2}$. Second, the use of unvalidated outcome measurements in association studies, such as parent or teacher-filled questionnaires on Likert scales ${ }^{3}$, is problematic. This approach applies to many of the underlying studies, and these questionnaires are neither developed nor validated for the purpose and context in which they are used. Third, the authors do not account for hereditary aspects of neurodevelopment, autism spectrum disorders (ASD) and attention deficit hyperactivity disorder (ADHD), and the ensuing importance of this as a confounder ${ }^{4}$. Fourth, on urogenital development, the underlying studies are unconvincing. A central study on APAP and cryptorchidism cited by the authors de facto reported an overall null association between APAP exposure and risk of cryptorchidism (adjusted OR 1.3, 95\% CI 0.70-2.6) ${ }^{5}$. In the pivotal study on anogenital distance (AGD) and APAP exposure, there was no difference in AGD between boys exposed to APAP and boys not exposed to APAP, as per the authors themselves: "Maternal use of paracetamol was not significantly associated with AGD in boys after adjustment." ${ }^{\text {. }}$. This null association has recently been confirmed ${ }^{7}$. Fifth, confirmation bias is present in Bauer et al. ${ }^{1}$; there is uncritical appraisal of studies that support the narrative of the authors. A study from the Boston Birth Cohort is assigned much weight by the authors to substantiate APAP exposure using umbilical cord plasma concentrations and correlating this exposure to childhood ADHD, ASD and other neurodevelopmental diagnoses ${ }^{8}$. We believe the external and internal validity of this study is compromised. APAP or metabolites were detected in every one of the 996 umbilical cord samples. Among the 996 children, a surprisingly large proportion were diagnosed with ADHD or ASD (37\%), and only $33 \%$ of children had no 'developmental disability' diagnosis. Finally, although preclinical data might support some mechanistic aspects of a hypothesis, it cannot be summoned to support a clinical recommendation.

The Scientific Committee members of the European Network of Teratology Information Services (ENTIS) - the authors of this Correspondence - do not share the position of Bauer et al. and believe that the supporting evidence brought forward is weak, inconsistent and to a large extent methodologically inadequate. The media headlines and social media reactions were predictable. Consequently, we believe that the Consensus Statement will promote unwarranted uncertainty, fear and guilt among pregnant women. It might also result in use of less safe alternatives, such as nonsteroidal anti-inflammatory drugs, during pregnancy.

There is a reply to this letter by Bauer, A. Z. et al. Nat Rev. Endocrinol. https://doi.org/ 10.1038/s41574-021-00610-1 (2021).

Per Damkier iD ${ }^{1,2}{ }^{\otimes}$, Brian Cleary ${ }^{3}$ Corinna Weber-Schoendorfer $r^{4}$, Svetlana Shechtman ${ }^{5}$, Matteo Cassina ${ }^{6}$, Alice Panchaud 7,8 and Orna Diav-Cirtin ${ }^{5,9}$

'Department of Clinical Biochemistry \& Pharmacology, Odense University Hospital, Odense, Denmark. ${ }^{2}$ Department of Clinical Research, University of Southern Denmark, Odense, Denmark. ${ }^{3}$ School of Pharmacy and Biomolecular Sciences, Royal College of Surgeons in Ireland. The Rotunda Hospital, Dublin, Ireland.

${ }^{4}$ Charité - Universitätsmedizin Berlin,

Pharmakovigilanzzentrum Embryonaltoxikologie, Institut für Klinische Pharmakologie und Toxikologie, Berlin, Germany.

${ }^{5}$ The Israeli Teratology Information Service, Ministry of Health, Jerusalem, Israel.

${ }^{6}$ Clinical Genetics Unit, Department of Women's and Children's Health, University of Padova, Padova, Italy. ${ }^{7}$ Institute of Primary Health Care (BIHAM), University of Bern, Bern, Switzerland. ${ }^{8}$ Service of Pharmacy, Lausanne University Hospital and University of Lausanne, Lausanne, Switzerland. ${ }^{9}$ The Hebrew University Hadassah Medical School, Jerusalem, Israel. 凶e-mail:pdamkier@health.sdu.dk https://doi.org/10.1038/s41574-021-00605-y

1. Bauer, A. Z. et al. Paracetamol use during pregnancy a call for precautionary action. Nat. Rev. Endocrinol. 17, 757-766 (2021)

2. Hernán, M. A. Causal Inferences from Observational Data https://www.hsph.harvard.edu/miguel-hernan/ research/causal-inference-from-observational-data/

3. Damkier, P., Pottegård, A., dePont Christensen, R. \& Hallas, J. Annotations and reflections: pregnancy and paracetamol: methodological considerations on the study of associations between in utero exposure to drugs and childhood neurodevelopment. Basic Clin. Pharmacol. Toxicol. 116, 2-5 (2015).

4. Masarwa, R., Platt, R. W. \& Filion, K. B. Acetaminophen use during pregnancy and the risk of attention deficit hyperactivity disorder: A causal association or bias? Paediatr. Perinat. Epidemiol. 34 , 309-317 (2020)

5. Kristensen, D. M. et al. Intrauterine exposure to mild analgesics is a risk factor for development of male reproductive disorders in human and rat. Hum. Reprod. 26, 235-244 (2011).

6. Lind, D. V. et al. Maternal use of mild analgesics during pregnancy associated with reduced anogenital distance in sons: a cohort study of 1027 mother-child pairs. Hum. Reprod. 32, 223-231 (2017).

7. Navarro-Lafuente, F. et al. Is maternal use of paracetamol during pregnancy associated with anogenital distance in male newborns? The results from the NELA birth cohort. Int. J. Environ. Res. Public Health 18, 6338 (2021).

8. Ji, Y. et al. Association of cord plasma biomarkers of in utero acetaminophen exposure with risk of attention-deficit/hyperactivity disorder and autism spectrum disorder in childhood. JAMA Psychiatry 77 180-189 (2020).

\section{Competing interests}

The authors declare no competing interests. 